\title{
The role of selenium supplementation in cardiovascular disease prevention: an in vitro study to identify the molecular mechanism(s)
}

\author{
D. Leighton, M. Goua, E. Dolan, K. Burgess and G. Bermano \\ Institute for Health and Wellbeing Research, Robert Gordon University, Aberdeen AB10 7 GJ, UK
}

\section{This abstract was awarded the student prize.}

Obesity is a worldwide epidemic, with two thirds of the UK population either overweight or obese (body mass index (BMI) 25-29.9 and $>30 \mathrm{~kg} / \mathrm{m}^{2}$ respectively $)^{(1)}$. Obesity is characterised by systemic oxidative stress (OS), which itself results from chronically high levels of reactive oxidative species (ROS) and reduced antioxidant status. OS is considered to play a key role in cardiovascular disease (CVD) development by initiating atherosclerosis ${ }^{(2)}$. In particular, increased monocyte ROS generation instigates atherosclerotic plaque formation by increasing the recruitment, binding and transmigration of monocytes across arterial endothelial cells into the arterial wall. An increased intake of dietary antioxidants or up-regulation of endogenous antioxidant enzymes may counteract this OS state and therefore lower CVD risk.

Selenium is an essential dietary micronutrient which is incorporated within the catalytic site of endogenous antioxidant Glutathione Peroxidase (GPx) enzymes and protects cells from OS and consequent cell damage. There is, however, a lack of knowledge concerning both the effect of selenium supplementation in an OS state representative of sedentary overweight/obese individuals and the mechanisms involved. The aim of this work was to investigate the effect of selenium supplementation in modulating, in vitro, monocyte cell viability, ROS generation and antioxidant enzymes gene expression (GPx1/GPx4) when under OS.

U937 monocyte cells were either supplemented, or not with sodium selenite $\left(\mathrm{Na}_{2} \mathrm{SeO}_{3} ; 100 \mathrm{nM}\right.$ or $\left.200 \mathrm{nM}\right)$ and cultured for 48 hours at $37^{\circ} \mathrm{C}$. Paraquat $(1 \mathrm{mM})$ and S-Nitroso-N-acetyl-DL-penicillamine $(0.7 \mathrm{mM})$ (SNAP) were added to cells overnight to induce OS. Cell viability was assessed via MTS (3-(4,5-dimethylthiazol-2-yl)-5-(3-carboxymethoxyphenyl)-2-(4-sulfophenyl)-2H-tetrazolium) assay, ROS generation was determined by Flow Cytometry using CM- ${ }_{2}$ DC-FDA, while GPx1/GPx4 gene expression was quantified by semi-quantitative reverse transcription polymerase chain reaction (RT-PCR).

PQ/SNAP treatment significantly reduced U937 cell viability and increased ROS generation compared to untreated control U937 cells; confirming the induction of OS. Supplementation with $100 \mathrm{nM} \mathrm{Na} 2 \mathrm{SeO}_{3}$, before PQ/SNAP treatment, significantly increased cell viability by $33 \%(\mathrm{p}<0.001)$ when compared with PQ/SNAP treated cells. Conversely, $200 \mathrm{nM} \mathrm{Na}_{2} \mathrm{SeO}_{3}$ supplementation did not significantly improve cell viability when compared to PQ/SNAP treated cells $(\mathrm{p}>0.05)$. Furthermore, $100 \mathrm{nM} \mathrm{Na}_{2} \mathrm{SeO}_{3}$ supplementation significantly reduced ROS generation by $32 \%(\mathrm{p}<0.001)$ in cells treated with PQ/SNAP. Correspondingly, this increased GPx1 by $146 \%(\mathrm{p}<0.01)$ and GPx4 gene expression by $77 \%(\mathrm{p}<0.05)$, when compared to un-supplemented PQ/SNAP treated cells.

This study indicates that selenium supplementation may be effective in counteracting the detrimental effects of OS by significantly increasing the expression of antioxidant genes; reinforcing endogenous antioxidant protection to quench ROS generation more effectively and improve cell viability. This preliminary data, in monocyte cells, indicates that GPx1 gene expression is affected considerably more by both selenium supplementation and OS when compared with GPx4. This suggests GPx1 may play a more important role in CVD prevention, with regards to selenium supplementation in overweight/obese individuals.

1. Health Survey for England (2011) (London: The Stationery Office).

2. Wadley AJ, Veldhuijzen van Zanten JJ, Aldred S (2013) AGE 35, 705-718.

3. Vendrov AE, Hakim ZS, Madamanchi NR, et al. (2007) Arterioscler Thromb Vasc Biol 27, 2714-2721. 